\title{
Recurrent oculogyric crisis and retrocollis after re-exposure with a low dose of olanzapine
}

Introduction of atypical antipsychotics has led to a decrease in acute dystonic reactions. The antiserotonergic and antimuscarinic activity of olanzapine may limit the development of extrapyramidal syndromes (EPS). Because of low propensity to cause EPS, olanzapine has been used successfully in cases with tardive dystonia. ${ }^{[1]}$ Previous studies also concluded that there were either rare or no dystonic reactions with olanzapine therapy. ${ }^{[2,3]}$ Moreover, one study observed no dystonic reaction with olanzapine even in higher doses in their acute phase of double blind olanzapine trial. ${ }^{[4]}$ Similarly, another study observed that despite the relatively high dose of olanzapine used, there were no signs of parkinsonism, akathisia, dystonia and none of their patients required anticholinergic medication. ${ }^{[5]}$ In contrary to these findings, I wish to report a case of olanzapine-induced dystonia in an adolescent girl.

The patient was a 14 year-old girl, from a lower socioeconomic background with no family history of any neurologic or psychiatric illness. Her personal history revealed that at the age of 2 , she suffered from encephalitis and subsequently had delayed developmental milestones. When she was brought to the OPD, 2 months ago, she was found to be aggressive, disruptive, and showed disinhibited behavior with disturbed biologic functioning including poor self care.

She was advised $2.5 \mathrm{mg} /$ day of olanzapine for her behavioral problems. With a single dose of olanzapine, she developed severe dystonic reaction, in the form of oculogyric crisis with retrocollis within 4 hours of taking olanzapine. She had sustained upward deviation of both eyeballs. Her neck was deviated to one side with stiffness in the neck muscles. She showed dramatic response to an intravenous injection of promethazine $50 \mathrm{mg}$. She was subsequently put on Lorazepam $1 \mathrm{mg} /$ day. She was investigated for the organic cause of dystonia, but her investigations, including the CT scan of the head and serum calcium levels did not reveal any abnormality.

During her follow-up visit after 15 days, she was re-exposed to the same dose of olanzapine and she again exhibited a similar kind of dystonic reaction within 10 hours of a single dose of olanzapine. Her dystonic reaction was again treated with intravenous injection of promethazine $50 \mathrm{mg}$. Later on, her behavioral problem was controlled with risperidone $2 \mathrm{mg}$ and trihexiphenidyl $2 \mathrm{mg} /$ day without any recurrence of dystonia.

Compared with typical antipsychotics, olanzapine has a greater affinity for serotonin $5-\mathrm{HT}_{2 \mathrm{~A}}$ than dopamine $\mathrm{D}_{2}$ receptors. Olanzapine is thought to have a preferential action at mesolimbic over nigrostriatal dopaminergic pathways and thus are associated with a very low incidence of extrapyramidal side effects than observed with typical antipsychotic drugs. In the Indian literature, reports on atypical antipsychotic-induced dystonia are scanty. ${ }^{[6]}$ To our knowledge, this is the first case report of recurrence of olanzapine-induced dystonia in the existing literature. This is not a coincidence finding because recurrence of dystonia was observed after re-exposing the patient with the same dose of olanzapine.

Oculogyric crisis with retrocollis can occur in a medical disorder such as encephalitis. ${ }^{17]}$ However, in this case, there was a definite temporal correlation between the onset of dystonic reaction and exposure to olanzapine. Apart from dopamine, other neurotransmitters, particularly serotonin has been proposed to play a role in acute dystonia but exact pathophysiology of atypical antipsychotic drug-induced dystonia is not known. ${ }^{[7]}$ This case report shows that even atypical antipsychotics such as olanzapine can induce dystonia in a predisposed individual, particularly in young children with history of central nervous system dysfunction. It also suggests that past history of dystonic reactions with atypical antipsychotics are potent predictors of developing dystonia when re-exposure to the same drug occurs.

\section{D.N. Mendhekar Department of Psychiatry, G.B. Pant Hospital, New Delhi \\ E-mail: drdnm@vsnl.net}

\section{References}

1. Jaffe ME, Simpson Gm. Reduction of tardive dystonia with olanzapine. Am J Psychiatry 1999;156:2016.

2. Gomez JC, The Safety of Olanzapine compared with other anti-psychotic drugs: $\mathrm{J}$ Clin Psychiatry 2001;62:828-9.

3. Raja M, Azzoni A. Novel anti-psychotics and acute dystonic reactions. Int J Neuropsychopharmacol 2001;4:393-7.

4. Beasley CM Jr, Tollefson GD, Tran PV, Satterlee W, Sanger T, Hamilton S. Olanzapine versus placebo and haloperidol: Acute phase results of the North American double-blind olanzapine trial. Neuropsycholopharmacol 1996;14:11123.

5. Martin J, Gomez JC, Garcia-Bernardo E, Cuesta M, Alvarez E, Gurpegui M. Olanzapine in treatment- refractory schizophrenia: Results of an open-label study. The spanish group for the study of olanzapine in treatment-refractory schizophrenia. J Clin Psychiatry 1997;58:479-83.

6. Bhana N, Foster RH, Onley R, Plosker GL. Olanzapine: An updated review. Drugs 2001;61:111-61.

7. Benjamin S. Oculogyric crisis in movement disorders in neurology and neuropsychiatry. In: Joseph A, Young R, editors. England: Blackwell Science; 1999. 